EGU2020-4053, updated on 10 Jun 2021

https://doi.org/10.5194/egusphere-egu2020-4053

EGU General Assembly 2020

(c) Author(s) 2021. This work is distributed under

the Creative Commons Attribution 4.0 License.

\title{
Study of the distribution of Rare Earth Elements in soil and in Vitis Vinifera L.cv Cannonau in two different regions
}

\author{
Salvatore Pepi ${ }^{1}$, Valeria Medoro ${ }^{1}$, and the Giulia Piroddi, Elena Marrocchino, Carmela Vaccaro ${ }^{*}$ \\ ${ }^{1}$ Università di Ferrara, Dipartimento di Fisica e Scienze della Terra, Ferrara, Italy (ppesvt@unife.it) \\ *A full list of authors appears at the end of the abstract
}

Vitis vinifera L. cultivar "Cannonau" (Magnoliopsida Vitaceae) has been grown for years in the Italian regions to produce a fine wine, with Controlled Designation of Origin (DOC) and Denomination of Controlled and Guaranteed Origin, (DOCG). The International Organization of Vine and Wine (OIV) defined the "terroir" as "a concept which refers to a specific area in which the interactions between the physical and biological environment and applied vitivinicultural practices develops. Whereas, from a geological point of view, the terroir has been defined as the geochemistry of soil, surface and ground water. Recent studies, regarding vitis vinifera, based on geochemical characterization have clearly shown the connection among geological origin, vineyard soil and grape berries. Another way to trace geographical origin can be through the identification of Rare Earth Elements (REEs) in the soil-plant system. However, the study of REEs is also important to define the petrological characterization and the relations between soil and plants.

We evaluated the relationship among the concentrations of rare earth elements (REE) in soil and in "Cannonau" grape berries in vineyards belonging to two different vineyards located in the valleys Pardu and Pelau in Sardinia (Italy) and one in Susegana in the Veneto Region (Northern Italy). The concentration of REE in samples of soil and juice or solid residues of grape berries was determined by inductively coupled plasma mass spectrometry (ICP-MS) and the data were elaborated with multivariate statistics (Linear Discrimination Analysis). The concentration of REEs in soil and grape berry samples allowed an identification of each locality examined. Moreover, the geochemical and statistical analyses allowed to discriminate the vineyard soils and grape berries according to geolithological characteristics of each area and to identify possible geochemical markers for the cultivar "Cannonau" .These markers, suitable as terroir fingerprintings, may be useful to avoid fraudulent use of the denomination label and falsification of the Made in Italy trademark.

Giulia Piroddi, Elena Marrocchino, Carmela Vaccaro: Università di Ferrara Giulia Piroddi email giulia.piroddi@student.unife.it Elena Marrocchino email Elena.marrocchino@unife.it Carmela Vaccaro email vcr@unife.it 